Service social

\title{
L’abus sexuel : discussion de la définition, éléments de diagnostic et de prévention
}

\section{Pierre Collart}

Volume 63, numéro 1, 2017

Interventions en matière d'agressions sexuelles

URI : https://id.erudit.org/iderudit/1040028ar

DOI : https://doi.org/10.7202/1040028ar

Aller au sommaire du numéro

Éditeur(s)

École de service social de l’Université Laval

ISSN

1708-1734 (numérique)

Découvrir la revue

Citer cet article

Collart, P. (2017). L’abus sexuel : discussion de la définition, éléments de diagnostic et de prévention. Service social, 63(1), 29-42.

https://doi.org/10.7202/1040028ar
Résumé de l'article

L'article aborde la définition de l'abus sexuel en développant la question du consentement, les actes composant l'abus sexuel et les processus impliqués dans l'abus sexuel, tant ce qui concerne les auteurs que les victimes. Dans un second temps, les conséquences psychologiques, psychosomatiques, physiques et physiologiques sont développées. Enfin, des éléments concernant la prévention des abus sexuels sont abordés. 


\title{
L'abus sexuel : discussion de la définition, éléments de diagnostic et de prévention ${ }^{1}$
}

\author{
COLLART, Pierre
}

\begin{abstract}
RÉSUMÉ
L'article aborde la définition de l'abus sexuel en développant la question du consentement, les actes composant l'abus sexuel et les processus impliqués dans l'abus sexuel, tant ce qui concerne les auteurs que les victimes. Dans un second temps, les conséquences psychologiques, psychosomatiques, physiques et physiologiques sont développées. Enfin, des éléments concernant la prévention des abus sexuels sont abordés.
\end{abstract}

Mots-clés : abus sexuel, consentement, conséquences, prévention.

\begin{abstract}
This article discusses the definition of sexual abuse by developing the issue of consent, the acts constituting the sexual abuse and the processes involved in sexual abuse, as regards the perpetrators and victims. Secondly, psychological, psychosomatic, physical and physiological consequences are developed. Finally, elements concerning sexual abuse prevention are discussed.
\end{abstract}

Keywords: sexual abuse, consent, consequences, prevention.

\section{INTRODUCTION}

Amnesty International relève sur base d'une récente enquête ${ }^{2}$ que $46 \%$ des personnes de leur échantillon, hommes et femmes, sont ou ont été victimes de violences sexuelles jugées "graves » au cours de leur vie. En ce qui concerne les femmes, $25 \%$ se sont fait et/ou se font encore harceler physiquement dans des lieux publics, $24,9 \%$ se sont fait et/ou se font imposer des relations sexuelles forcées par leur partenaire/conjoint, $13 \%$ se sont fait violer et/ou se font encore actuellement violer par un auteur autre que par leur partenaire ou conjoint et $7 \%$ se font et/ou se sont fait abuser sexuellement par un adulte alors qu'elles étaient encore mineures. D'autres chiffres font état d'attouchements sexuels subis avant l'âge de 18 ans par $9 \%$ des femmes et $3 \%$ des hommes en Belgique.

Au niveau européen, l'examen des études de prévalence de l'abus sexuel d'enfant mettent en évidence des taux de prévalence globale de 6 à $36 \%$ chez les filles et de 1 à $15 \%$ chez les garçons, tous âgés de moins de 16 ans, tandis que d'autres études indiquent des taux de viol de $0,9 \%$ pour les femmes et $0,6 \%$ pour les hommes, taux passant à $50 \%$ pour les femmes

\footnotetext{
${ }^{1}$ Paru dans Louvain Médical, 2015, 134 (8), hors série, P. 3-11.

2 Étude des opinions et des comportements de la population belge en matière de violences sexuelles, menée par Dedicated pour Amnesty International et SOS Viol. Sondage réalisé en janvier 2014 par internet sur un échantillon strictement représentatif de 2000 Belges âges de 18 à 75 ans ( 960 hommes et 1040 femmes).
} 
et $25 \%$ pour les hommes lorsque l'on utilise des définitions plus larges de l'abus sexuel d'enfant $^{3}$.

À eux seuls, ces chiffres montrent à quel point l'abus sexuel est fréquent. Et complexe. Il est illusoire, en quelques pages, d'en faire un état de lieux exhaustif. Nous nous limiterons donc ici à donner quelques pistes afin de définir l'abus sexuel, ainsi que quelques éléments de diagnostic et de prévention.

\section{Définir l'abus sexuel}

Il existe de multiples définitions de l'abus sexuel ${ }^{4}$. Nous proposons ici de le définir comme toute interaction sexuelle impliquant une / des personne(s) qui n'y consent(ent) pas. Cette définition extensive nous conduit à appréhender la représentation actuelle de l'abus sexuel en suivant trois axes : ce qui la détermine, les comportements qui la composent et les processus qui sont à l'œuvre.

\section{Le consentement}

Le critère central actuel pour une sexualité socialement non problématique est le consentement. II s'agit en effet de donner la primauté à l'acceptation - ou au refus - de l'individu à s'engager dans une activité sexuelle déterminée avec un ou des partenaires clairement identifié(s). Ce modèle actuel de sexualité se réfère à ce que Michel Foucault appelle le "dispositif de sexualité » selon lequel l'accord du partenaire est requis et l'outrepasser rend le rapport sexuel illégitime ${ }^{5}$. Les conséquences en sont également que la sexualité est devenue une affaire privée, individualisée et susceptible de sanction sociale s'il y a non-respect du non-consentement.

II est dès lors nécessaire de préciser les critères de validité du consentement sexuel. D'après la formule consacrée, il doit être «libre et éclairé ». Ce qui signifie d'une part qu'il doit être obtenu sans contrainte. L'OMS inclut d'ailleurs cette dimension du consentement dans sa définition de la santé sexuelle, en précisant que celle-ci requiert: " une approche respectueuse de la sexualité et des relations sexuelles, ainsi que la possibilité d'avoir des expériences sexuelles (...) libres de toute coercition, discrimination ou violence » (OMS, 2015). D'autre part, la personne qui consent doit être au clair avec ce à quoi elle s'engage, ce qui implique « la capacité à comprendre et évaluer les risques, les responsabilités, les issues et les impacts des actes sexuels, et de s'en abstenir lorsque c'est approprié » (Miner, 2001). II y

\footnotetext{
${ }^{3}$ À ce propos, Leventhal explique la diminution de $31 \%$ de cas établis d'abus sexuels d'enfants entre 1992 et 1998 aux USA (39\% entre 1992 et 1999 selon Jones, Finkelhor et Kopiec) par des différences méthodologiques, parmi lesquelles il retient la définition de l'abus sexuel d'enfant et l'âge utilisé pour définir l'enfance. La diminution des situations d'abus sexuels rapportées ne reflèterait donc pas le véritable changement dans l'incidence de l'abus sexuel, mais plutôt les évolutions dans les critères utilisés par les services de protection de l'enfance pour accepter et valider un cas d'abus sexuel d'enfant (Collart). Voir également à ce propos Lalor et McElvaney.

${ }^{4}$ Born et al. précisent à ce propos que « toute définition varie en fonction de l'utilisation concrète qui en est faite (les perspectives du chercheur, du clinicien, de l'intervenant social, du juriste et des services de l'ordre ne sont pas forcément superposables) et dépend du contexte social, culturel et historique ».

${ }^{5}$ Ce dispositif de sexualité s'opposant et se substituant progressivement au « dispositif d'alliance » basé sur une sexualité statutaire où le permis et l'interdit étaient fonction du statut des partenaires, où seuls les rapports sexuels entre époux étaient légitimes (Foucault; Marquet). Précisons à ce propos qu'en Belgique, ce n'est qu'en 1989 que le viol entre époux a commencé à avoir une existence légale. Jusqu'à cette date, le conjoint était supposé consentir aux relations sexuelles, du fait de son statut matrimonial.
} 
aura donc abus sexuel lorsque l'une et/ou l'autre de ces conditions ne sera pas rencontrée dans l'interaction sexuelle.

Ces caractéristiques du consentement déterminent une catégorie particulière d'individus qui n'ont a priori pas la capacité d'émettre un consentement valable en raison à la fois de leur immaturité et de leur dépendance à l'égard de l'adulte : les enfants. Ce qui, en corollaire, considère toute interaction sexuelle entre un adulte et un enfant comme étant un abus sexuel et en attribue automatiquement la responsabilité à l'adulte.

Une seconde conséquence de cette définition concerne les subtilités du consentement. Bien que son principe paraisse simple, ses déclinaisons culturelles, légales, interactionnelles et individuelles rendent parfois son appréciation beaucoup plus subtile, voire complexe ${ }^{6}$ : à quoi consent-on lorsque l'on consent? Quelles sont la portée et la durée de validité du consentement? Idéalement, les protagonistes d'une interaction sexuelle devraient pouvoir mettre leurs limites voire exprimer leur refus de tel ou tel acte à tout moment de cette interaction, ce qui devrait être entendu et respecté par leur partenaire. Si le consentement est donc un accord de s'engager dans une activité se déroulant dans l'instant, il devrait donc pouvoir être revu à tout moment, par chaque protagoniste, en fonction de critères qui lui sont propres, et n'implique pas un accord pour les interactions futures ${ }^{7}$. On imagine dès lors bien à quel point ce consentement peut parfois être au centre de pressions et de jeux relationnels dans les dynamiques de couple, par exemple.

\section{Les actes sexuellement abusifs}

Les comportements impliqués dans les abus sexuels - il s'agit de notre second axe d'approche - se déploient dans une pluralité de contextes distincts et se déclinent en une grande variété d'actes.

Les contextes concernés recouvrent aussi bien le harcèlement sexuel au travail ${ }^{8}$ que les viols en situation de guerre ${ }^{9}$, en passant, ce qui est le cas le plus fréquent dans le contexte belge actuel, par les abus intrafamiliaux (les plus fréquents, qu'il s'agisse des abus sexuels d'enfants que des abus sexuels entre conjoints) et extrafamiliaux, ces derniers recouvrant les abus commis par des personnes connues ou par des inconnus.

En ce qui concerne les actes qui matérialisent l'abus sexuel, ils ne se limitent pas au seul contact des zones génitales, mais prennent également d'autres formes, dont les classifications varient selon les auteurs. L'abus sexuel inclut donc tous les actes orientés vers l'intimité corporelle de la victime, qui agressent sa sphère sensorielle et/ou qui utilisent son corps. Ils

\footnotetext{
${ }^{6}$ II s'agit là le l'argument du Congrès international francophone sur l'agression sexuelle qui s'est tenu en 2015 à Charleroi : le constat que l'abus sexuel est une réalité complexe, multiforme et multidimensionnelle, demandant de la part des intervenants une gamme de réponses adaptées.

${ }^{7}$ A l'instar de la loi 967 de Californie visant à combattre les agressions sexuelles sur les campus (concernant une femme sur cinq), qui prévoit que les partenaires sexuels doivent donner «leur accord explicite, conscient et volontaire » avant toute relation sexuelle. "L'accord explicite ne peut être donné par quelqu'un d'endormi, d'inconscient », ou s'il ou si elle est « sous l'influence de drogues, d'alcool ou de médicaments ».

${ }^{8}$ La loi sur le bien-être au travail (1996) définit le harcèlement sexuel au travail comme « tout comportement non désiré verbal, non verbal ou corporel à connotation sexuelle, ayant pour objet ou pour effet de porter atteinte à la dignité d'une personne ou de créer un environnement intimidant, hostile, dégradant, humiliant ou offensant ».

${ }^{9}$ Par exemple, on estime à des centaines de milliers les victimes de viols et de violences sexuelles en Europe lors de la seconde guerre mondiale, à plus de 80 \% de femmes violées lors des conflits au Kivu entre 1996 et 2003. Commission Justice et Paix.
} 
comprennent « des formes verbales et non verbales, gestuelles, visuelles, exhibitionnistes, voyeuristes, masturbations manuelles, fellations, ..., jusqu'aux relations sexuelles complètes, avec pénétration orale, anale ou vaginale » (Haesevoets, 1997), par un doigt, le sexe, un objet. Gosset et al. par exemple distinguent deux formes d'abus sexuels :

1. Les actes agressant la sphère sensorielle de la victime :

- conversations ou appel téléphonique obscène, « chat » sexuel;

- $\quad$ présentation forcée d'images pornographiques (photos, vidéos);

- $\quad$ exhibition d'organes sexuels.

2. Les actes qui utilisent le corps :

- $\quad$ attouchements sexuels ou masturbation forcée;

- toilette intime imposée;

- $\quad$ relations sexuelles sous la contrainte (fellation, sodomie, rapport vaginal);

- participation active à des scènes pornographiques;

- incitation à la prostitution.

Ces auteurs ajoutent également à cette liste les mutilations sexuelles chez la fillette (excision, infibulation, clitoridectomie).

D'autres auteurs, comme May ${ }^{10}$, proposent une classification tripartite, dans laquelle l'abus sexuel dépasse largement la notion d'une «implication des parties génitales » : à côté des abus sans attouchements physiques (incluant l'exhibitionnisme, les téléphones obscènes et les verbalisations abusives) et des abus impliquant un contact avec le corps de la victime (comprenant les caresses, les stimulations génitales et orales, les relations sexuelles complètes ou leur équivalent), ils développent une troisième catégorie caractérisée principalement par la violence, dans laquelle on retrouve par exemple le viol, les blessures physiques et le meurtre sadique ${ }^{11}$. II faut également noter que ces comportements abusifs peuvent apparaître entre un adulte et un enfant ou entre pairs ${ }^{12}$, et dans un contexte intra ou extrafamilial ${ }^{13}$.

Ces dernières années, le développement des réseaux sociaux sur Internet a favorisé l'émergence de nouveaux comportements sexuellement abusifs ayant comme victimes principales les adolescents et les jeunes adultes : la cybermanipulation, la cyberprédation et l'autoproduction, par des personnes mineures, de matériel érotique ou pornographique ${ }^{14}$. Bien

\footnotetext{
${ }^{10}$ May, 1977, cité par Van Gijseghem : 16.

${ }^{11}$ De telles classifications pouvant toutefois être discutées, certaines approches arguant en effet que tout abus sexuel est d'abord une interaction violente, la dimension sexuelle étant dans tous les cas secondaire.

12 Born et al., Burnay. Dans le cas précis d'un abus sexuel entre pairs, on se réfère alors uniquement à la question du consentement, et pas à la question d'une distribution inégalitaire du pouvoir (cfr. Finkelhor).

${ }^{13}$ Born et al., Fischer et al., Miller-Perrin \& Perrin, Schneider.

${ }^{14}$ Ces nouvelles pratiques abusives ont été présentées par Michel Dorais (Université Laval, Québec), au $8^{e}$ congrès international francophone sur l'agression sexuelle, en juin 2015. Selon lui, la cybermanipulation à des fins sexuelles, pratique la plus courante (95\% des cas), est agie par un cybermanipulateur qui ne ment pas (ou peu) sur son âge ou sur son identité: il se présente comme un adulte qui peut être un " confident » ou " mentor » sur le plan affectif. La cyberprédation est agie par un cyberprédateur qui ment sur son âge et sur ses intentions, présentées comme amicales, « entre jeunes ». Le cyberprédateur vise à obtenir des rencontres afin d'avoir alors des contacts sexuels. Quant à l'auto-production de matériel érotique ou pornographique, elle se fait parfois dans un contexte amoureux (du moins du point de vue de la victime).
} 
que ces derniers puissent trouver leur place dans les classifications développées ci-avant, ils sont toutefois significatifs de l'adaptation des comportements sexuels abusifs aux nouvelles technologies de l'information et de la communication.

\section{Les processus des passages à l'acte sexuellement abusifs}

Le troisième axe d'analyse de l'abus sexuel porte sur les processus à l'œuvre dans le passage à l'acte.

En ce qui concerne l'auteur, c'est l'hétérogénéité des situations qui prévaut. J. Proulx précise à ce propos que " le passage à l'acte abusif est déterminé par des variables structurales, situationnelles et relatives au modus operandi ».

L'analyse des variables structurales stables concerne l'organisation de pensée, d'émotion et de comportements. À propos de ces caractéristiques de personnalité, la recherche d'un profil de personnalité unique, propre aux agresseurs sexuels ne concorde pas avec la diversité de ces auteurs. Toutefois, l'attachement de type insécure est une caractéristique spécifique des agresseurs sexuels.

Les facteurs situationnels, eux, concernent des aspects circonstanciels (perte d'un emploi, décès, séparation, divorce...) et désinhibiteurs (alcool, drogues, émotions négatives - colère, rage, haine... - pornographie...) et accroissent ponctuellement le risque de répétition.

Quant aux caractéristiques du mode opératoire, elles sont multiples (stratégies, type de l'acte abusif, lien agresseur/victime, réaction de la victime...) et sont donc à comprendre dans une logique interactionnelle.

Les différentes approches théoriques de la psychologie mettent par ailleurs en évidence des hypothèses explicatives distinctes que l'on peut considérer comme complémentaires à l'épreuve de la clinique. Ainsi, la psychanalyse a dans un premier temps émis l'hypothèse de la perversion afin d'expliquer le passage à l'acte sexuel abusif, pour ensuite élargir l'explication aux différentes structures de personnalité et proposer des processus psychiques distincts au passage à l'acte (manque de contrôle de la pulsion, intolérance à la frustration, fusion, séduction, double, fétichisation, toute-puissance, support-groupal). L'approche systémique, pour sa part, met en évidence les caractéristiques relationnelles des relations sexuelles abusives (essentiellement incestueuses), en termes de complémentarité rigide, de domination de l'enfant au bénéfice de l'adulte, d'imposture, de perversion de la dialectique autoritéresponsabilité, de relation hors-la-loi, hors contexte, hors contrôle, voire d'emprise. Quant à la lecture cognitivo-comportementale, elle évoque plutôt d'une part les contenus des apprentissages et conditionnements de l'individu, ainsi que les éventuels déficits d'habiletés susceptibles de provoquer un stress que l'individu tentera de gérer par le comportement sexuel abusif.

Les motivations de l'auteur d'abus sont donc diverses et influencent le mode opératoire du passage à l'acte, où le degré de violence physique accompagnant l'abus sexuel peut être très variable, de pratiquement nul lorsque l'auteur évoque des interactions « amoureuses » ou se positionne en tant que victime d'un enfant qui l'a séduit, par exemple, à très élevé lorsqu'il s'agit d'une démarche de consommation sexuelle de l'autre ou encore lorsque le degré d'agressivité de l'auteur envers la victime est élevé. Mais à côté de cette violence physique 
potentiellement présente, il faut également considérer la présence de la violence psychologique, omniprésente. Dans les abus sexuels d'enfants, celle-ci a pour principal objet d'imposer le silence, le secret étant la caractéristique principale de ces abus. Cette violence psychologique est multiforme, prenant l'aspect de la séduction, de la gentillesse, de « l'achat » du silence par des cadeaux, ou encore de menaces de séparation, de punition, voire de mort.

L'étude des violeurs de personnes adultes met également en évidence plusieurs motivations, qui se traduisent par des modes opératoires distincts. Ainsi par exemple, Knight \& Prentky distinguent ainsi le violeur opportuniste, qui agit impulsivement, utilise la force nécessaire pour soumettre sa victime qu'il traite comme un objet; le violeur présentant une «rage indifférenciée », impulsif et " gratuitement » violent même si la victime ne résiste pas et ne tirant pas d'excitation sexuelle de sa violence; le violeur à motivation sexuelle, sadique ou non; le violeur à motivation vindicative, dont la colère est exclusivement dirigée vers les femmes, qui pose des actes brutaux dans le but d'humilier et détruire la femme, le viol étant le moyen d'y arriver.

Pour ce qui concerne les victimes d'abus sexuel, des processus de victimisation ont également été mis en évidence, particulièrement lorsqu'il s'agit d'abus sexuels d'enfants s'étalant dans le temps. Ainsi, à titre d'exemple, Summit ${ }^{15}$, décrit le syndrome d'accomodation de l'enfant à l'abus sexuel, qui conduit la victime d'abus à considérer son sort comme une fatalité : l'enfant commence par s'engager en confiance dans une activité nouvelle pour lui avec un adulte. II devient plus ou moins rapidement perplexe, car il perçoit vaguement que quelque chose est anormal, qui lui procure des sensations qu'il ne comprend pas, qu'il souhaiterait arrêter, mais sans pouvoir le faire de peur de déplaire à l'adulte. Le secret s'installe alors autour de ces contacts, une «loi du silence » est instaurée. L'enfant est, à ce moment-là, totalement impuissant et partagé entre les deux positions opposées de rejet de l'adulte abuseur et d'affection envers ce même adulte qui lui montre parfois une affection normale. L'enfant évolue alors vers une phase de "coping ", de résignation aux contacts sexuels avec l'adulte. II peut par la suite - parfois beaucoup plus tard - révéler les faits, ce qui s'accompagnera d'une anxiété importante et d'un risque de rétractation si les dires de l'enfant sont mis en doute par son entourage.

Notons encore que lorsque la victime de l'abus sexuel est une personne adulte on retrouvera généralement chez elle des sentiments de culpabilité (la victime se reproche un acte répréhensible, sans nécessairement de lien avec la réalité des faits), de souillure (proche de la stigmatisation, consécutive à la transgression d'une norme ou d'un tabou) et de honte (basée sur l'expérience de l'indignité et de l'humiliation face à autrui).

\section{Le diagnostic de l'abus sexuel}

La mise en évidence de ces processus traumatogènes conduit tout naturellement à la question du diagnostic de l'abus sexuel.

À ce jour, les auteurs s'accordent sur le fait qu'il n'y a pas de signe pathognomonique de l'abus sexuel. Le clinicien se trouvera plutôt face à un ensemble plus ou moins étendu de symptômes

\footnotetext{
${ }^{15}$ Le modèle de Summit concerne essentiellement les situations d'inceste mais reste valable pour les abus sexuels extrafamiliaux.
} 
plus ou moins directement évocateurs d'abus sexuel, qu'il doit confronter à la parole de la victime et au contexte de la révélation.

Haesevoets propose un modèle général des symptômes qui évoquent la possibilité d'abus sexuel envers un enfant qui s'inspire de différentes classifications :

Les symptômes physiques ou physiologiques ${ }^{16}$ regroupent la possibilité de lésions des organes génitaux, hématomes de l'abdomen ou sur les zones péri-génitales; de douleurs dans les régions génitales, saignements, douleurs à la miction; de dilatation de l'urètre, de l'anus, du vagin; de présence de corps étrangers dans l'urètre, la vessie, le vagin ou l'anus; d'infection des voies urinaires; de présence d'une maladie sexuellement transmissible; de grossesse chez les adolescentes qui la cachent ou qui sont évasives quant à la paternité.

Les symptômes psychosomatiques regroupent la possibilité d'énurésie et d'encoprésie; de céphalées récidivantes; de douleurs abdominales; de crises de conversion hystérique; de troubles du sommeil; de troubles de la conduite alimentaire, d'anorexie, de boulimie.

Les symptômes psychologiques et comportementaux regroupent la possibilité d'inhibition, de traits dépressifs, d'anxiété, de tristesse; de manque de confiance en soi ou/et en autrui; de distorsion de la perception de soi, d'autodépréciation, de perte d'estime de soi, de négligence, de manque d'hygiène, de dénarcissisation; d'isolement par rapport aux sujets du même âge, de solitude, d'attitudes ou de conduites d'évitement; de préoccupations d'ordre sexuel; d'allusion à des questions d'ordre sexuel, à travers des jeux ou des dessins; de conduite sexuelle inappropriée ou masturbation excessive; de sexualisation de la relation humaine; de troubles $d u$ caractère, d'agitation, d'agressivité, de sauts d'humeur, d'acting out violent; de confusion des sentiments et d' ambivalence; de sentiment d'être victime et d'attitude de " victimisation "; de troubles de la conduite sociale, de conduites antisociales; de troubles d'apprentissage (dyscalculie, dysorthographie) et de la concentration; de troubles intellectuels; de chute du rendement scolaire, d'apathie scolaire, de difficultés d'apprentissage, de trouble du raisonnement logico-mathématique.

Chez les enfants plus âgés et les adolescents, les troubles psychoaffectifs peuvent se traduire par une culpabilité liée à un certain niveau d' acceptation ou de participation à I' activité abusive; une culpabilité liée aux sentiments d'hostilité ou à l'agressivité à l'égard du parent non-abuseur; des troubles de l'identité ou de la personnalité; des fugues; des auto-lésions et tentatives de suicide; des conduites automutilantes; des conduites addictives ou toxicomaniaques; une faillite de la formation scolaire et professionnelle; de l'inadaptation sexuelle, frigidité, dysfonction orgasmique, confusion dans l'orientation sexuelle, homosexualité coupable ou mal vécue; des pratiques de promiscuité sexuelle, de prostitution, de masochisme ou de victimisation sexuelle; des conduites délinquantes diverses.

Ces données sont largement confirmées par la revue de la littérature de Tyler : par rapport aux adolescents qui ne rapportent pas d'épisodes d'abus sexuels, les adolescents qui ont été victimes d'abus sexuels durant l'enfance présentent une fréquence plus élevée de

\footnotetext{
${ }^{16}$ Voir à ce propos Adams.
} 
dépressions, d'idées suicidaires et de consommation d'alcool et de drogue, de comportements sexuels à risque et de grossesse, de prostitution, de nouvelle victimisation sexuelle, de fugues, de troubles comportementaux tels que l'isolement social, des conduites socialement déviantes, des conduites agressives. Le risque de présenter un syndrome de stress posttraumatique (selon les critères du DSM IV) est également bien présent, surtout lorsque l'abus s'est étalé sur une longue période, ou que l'enfant a été menacé ou contraint physiquement. De manière générale, d'ailleurs, Tyler relève que la sévérité de l'abus, l'usage de la force et le type de lien entre la victime et l'abuseur (parent, connaissance, inconnu) sont des éléments déterminants pour l'apparition des effets de l'abus ${ }^{17}$.

À côté de ces différents symptômes, le récit de la victime est déterminant. On y trouvera sa perception des événements, les éléments de compréhension de la violence psychologie et/ou physique subie, les étapes franchies afin d'en arriver au dévoilement. Recueillir cette parole nécessite de porter une attention à la manière dont s'opère ce recueil et à la validité de cette parole. Plusieurs procédures et protocoles existent à ce sujet, qu'il s'agisse de recueil et de l'évaluation du récit de la victime dans un contexte clinique ou dans un contexte judiciaire et qu'il s'agisse d'une victime adulte ou mineure.

L'intérêt du recours à de tels protocoles de recueil des témoignages par rapport à un recueil hors protocole semble se confirmer. Ainsi pour ce qui concerne les enfants, Arnould souligne que l'utilisation d'un protocole (NICHD) ${ }^{18}$ " a incité les interviewers à utiliser un plus grand nombre d'invitations libres (...) et à limiter l'usage des questions dirigées, à option et suggestives (...). Le protocole a permis également de poser un moins grand nombre de questions (...) tout en permettant d'obtenir des détails intéressants de la part des enfants. (...). Quel que soit leur âge, [les enfants] ont notamment communiqué un plus grand nombre de détails lorsqu'ils ont été interrogés au moyen de cette procédure par rapport aux entretiens hors protocole».

Le contexte de production de cette parole, les influences auxquelles est exposée la victime sont par ailleurs des éléments justifiant le recours à des protocoles d'évaluation de la crédibilité du témoignage de l'enfant - voire également de l'adulte ${ }^{19}$. II faut en effet être conscient qu'à côté des révélations d'abus sexuels réels, les enfants - et les adultes - qui dévoilent de tels faits peuvent également être empêtrés dans des attitudes de fausses allégations, victimes par exemple de manipulation mentale dans des situations de conflits familiaux. La tâche du clinicien consiste ainsi dans tous les cas à poser le diagnostic correct de la situation qui se présente à lui pour proposer à la victime - de quelque atteinte qu'il soit - la prise en charge la plus adéquate.

\footnotetext{
17 Tyler pose toutefois une question récurrente lors de sa revue de littérature : quel type de relation peut-on supposer entre l'abus sexuel durant l'enfance et les comportements mis en évidence dans les différentes études. En effet, si des corrélations ont été mises en évidence, il reste à se demander si un lien de causalité peut être établi, et dans quel sens. Ainsi par exemple, les idées suicidaires sont-elles une conséquence des abus sexuels, ou les enfants dépressifs et suicidaires présentent-ils des risques plus élevés d'abus sexuels?

18 Lamb et al. Protocole du National Institute of Child Health and Human Development, entretien structuré basé essentiellement sur des questions ouvertes, dont une version française est disponible, organisé en cinq phases : Introduction, phase de contact, phase de transition, phase de rappel libre, phase de questionnement directif.

${ }^{19}$ Niveau et al. Le protocole le plus connu en cette matière est le SVA (Statement Reality Analysis). II se compose d'une interview semi-structurée, d'une échelle d'évaluation « Criteria based content analysis », ou CBCA, et d'une «Validity checklist» permettant de pondérer les résultats du score à l'échelle d'évaluation. Voir également Haesevoets.
} 


\section{Quelques mots à propos de la prévention}

La meilleure adéquation possible de la prise en charge implique de se soucier de la prévention de l'abus sexuel, tant en ce qui concerne l'auteur que la victime.

En matière de prévention primaire, c'est probablement très en amont que le travail essentiel doit être mené. Il s'agit en effet de penser une véritable éducation au respect de soi et des autres, qui consisterait à permettre à chacun de développer suffisamment de considération pour soi et pour l'autre que pour avoir la capacité d'émettre ses souhaits et ses limites, et d'entendre celle de l'autre. Cette démarche, bien entendu, dépasse le cadre strict de la sexualité pour englober l'ensemble des aspects du «soi » et du «vivre ensemble ». Elle devrait, pour être réellement efficace, débuter très tôt dans la vie de chacun.

La prévention primaire axée spécifiquement sur l'abus sexuel pose la question des programmes et campagnes de prévention à destination des enfants ou des adultes. Régulièrement, ceux-ci sont pointés comme étant inutiles, anxiogènes, voire toxiques en ce que, par exemple, ils responsabilisent la victime (enfant ou adulte) de l'abus qu'elle a subi. À ce propos, nous soumettons à la réflexion cette conclusion de Yakapa.be : " quand les adultes sont à l'écoute des questions de l'enfant au fur et à mesure qu'elles apparaissent et quand ils les initient à la vie en commun - ici et maintenant - de leur place d'adultes responsables et capables de parler, alors ils font œuvre de prévention. Animateurs, éducateurs, enseignants... n'ont pas besoin de programme clé sur porte pour un tel travail. II s'agit avant tout de tenir une place et de saisir les petits événements du quotidien, au fur et à mesure de leur surgissement » (Collectif Yakapa.be, 2013).

En ce qui concerne la prévention secondaire et tertiaire, à propos des auteurs d'abus sexuel, une réflexion sur la prise en charge doit se poursuivre. Leur «traitement social » actuel, impliquant à la fois la sanction et le soin, est une avancée majeure en Belgique depuis maintenant près de vingt ans. Des questions continuent toutefois à se poser, notamment en terme de modalités de la sanction. Les conditions d'emprisonnement tel qu'on les connaît actuellement ne permettent toujours pas que ce temps passé derrière les barreaux soit réellement mis à profit pour une évolution et/ou une reconstruction psychique de l'individu en vue de sa libération. Les pratiques cliniques doivent continuer à évoluer vers une prise en charge de la personne, dans sa globalité et sa complexité, recourant aux capacités et compétences de la personne elle-même pour développer des solutions visant à la fois les deux objectifs essentiels que sont la prévention de la répétition de l'abus et l'épanouissement personnel de l'auteur.

La prise en charge des victimes, au-delà des soins immédiats à leur apporter, doit pour sa part éviter la victimisation secondaire. Cela suppose que la victime doit pouvoir être accueillie, entendue et assurée qu'elle sera traitée avec dignité. D'un point de vue clinique, cela demande également un accompagnement dans la durée, qui puisse reconnaître la personne dans sa dimension de victime (avec les dimensions de culpabilité, de honte, de souillure) sans toutefois l'y enfermer, en l'accompagnant dans la mobilisation de ses compétences.

Le développement de telles «bonnes pratiques" implique également de favoriser la rencontre, le dialogue et la compréhension mutuelle entre les intervenants des différents champs disciplinaires impliqués : acteurs de la justice, des soins, intervenants sociaux... qui 
doivent par ailleurs encore parfois faire l'effort de sortir d'un certain clivage existant entre ceux qui prennent en charge les victimes et ceux qui traitent les auteurs.

Enfin, il ne faut pas faire l'impasse sur la responsabilité du monde politique dont dépend in fine la reconnaissance sociale du phénomène et la réponse à lui apporter en terme de financement et d'organisation des soins et des pratiques judiciaires à l'égard des auteurs et victimes d'abus sexuel.

\section{Conclusion}

L'abus sexuel est un phénomène complexe et ubiquitaire. Fondé dans sa définition actuelle sur le principe du consentement, il consacre la possibilité de l'individu de décider de son (non)engagement dans une activité sexuelle. Et identifie une catégorie de personnes qui ne peuvent valablement user de cette faculté : les enfants, qui de ce fait méritent de faire l'objet d'une protection particulière face aux interactions sexuelles dans lesquelles les engageraient des adultes et qui, dans tous les cas, seront considérées comme des abus sexuels.

On l'a vu également, l'abus sexuel est susceptible de causer à la victime de multiples séquelles, tant psychologiques que somatiques et fonctionnelles. Ces conséquences sont présentes à court terme, mais peuvent également, c'est régulièrement le cas, impacter la vie de la victime sur le long terme et dans plusieurs aspects de sa vie. Toutefois, rappelons-le, il y a des victimes d'abus sexuels qui s'en sortent et vont bien. La connaissance des processus de résilience nous montre que chacun a des ressources qui peuvent être mobilisées. C'est au clinicien à accompagner son patient, parfois au long cours, dans ce cheminement.

Exploiter les ressources positives de la personne est également une piste intéressante pour la prise en charge de l'auteur d'abus sexuel. S'occuper de l'abus sexuel ne peut en effet pas faire l'économie d'une préoccupation clinique et thérapeutique à l'égard des auteurs. On pourrait dire en effet "qu'il n'y a pas d'abuseur heureux », et que l'épanouissement de la personne qui, à un moment de sa vie, commet des actes d'abus sexuel est une démarche curative, mais également axée vers la prévention en ce qu'elle vise à prémunir contre la répétition des abus.

La prévention, qu'elle soit primaire, secondaire ou tertiaire est enfin le domaine où l'investissement sociétal doit porter. Les soins aux victimes et aux auteurs doivent être accompagnés d'une réflexion plus globale sur la manière dont le respect de soi et de l'autre doit être valorisé et développé. Cette réflexion doit être portée et mise en actes par les acteurs éducatifs, cliniques, judiciaires, politiques à leurs niveaux respectifs. Elle doit également être un moteur de nos actions en tant que parent, en tant qu'humain. Cela dépasse bien entendu la « seule » question de l'abus sexuel.

Pierre Collart

Docteur en sciences psychologiques UCL, ESFA

CHU de Charleroi, Hôpital Vincent Van Gogh, Belgique 


\section{RÉFÉRENCE}

Adams J.A. Medical Evaluation of Suspected Child Sexual Abuse; 2011 Update. Journal Of Child Sex Abuse, 2011, 20 : 588-605.

Arnould F. Interroger les enfants: une version française du protocole du NICHD. PsychoTémoins. Actualité de la recherche sur les témoignages en justice. http://psychotemoins.inist.fr/? Interroger-les-enfants-une-version Dernière mise à jour le 08.01.2014.

Balier C. Psychanalyse des comportements sexuels violents. PUF, Paris, 1996.

Born M., Delville J., Mercier M., Sand E.A., Beeckmans M. Les abus sexuels d'enfants. Interventions et représentations. Mardaga, Liège, 1996.

Burnay N. N'a-t-on pas oublié de dire... De l'amalgame de l'abus sexuel aux discours sur l'enfant. In Burnay N., Lannoy P., Panafit L. (dir.), La société indicible. La Belgique entre émotions, silence et parole, Ed. Luc Pire, Bruxelles, 1997 : 15-24.

Collart P. Abuseurs sexuels d'enfants et rapports à la norme. Étude par l'interactionnisme symbolique des logiques de rapports au cadre social normatif construites par des adultes impliqués dans des interactions sexuelles avec des enfants. Louvain-la-Neuve, UCL. 3 tomes. Thèse de doctorat en sciences psychologiques - orientation famille et sexualité. 2004.

Collart P. Les abuseurs sexuels d'enfants et la norme sociale. Académia-Bruylant, Louvain-laNeuve, 2005.

Collectif Yakapa.be. Faut-il prévenir les enfants des abus sexuels? Points de repère pour prévenir la maltraitance. Temps d'arrêt. Yakapa.be. 2013 : 105-107.

Commission Justice et Paix. Le viol comme tactique de guerre. Le cas de la République Démocratique du Congo. Analyse 2007, 7P.

Conseil des femmes francophones de Belgique. Protocoles de prise en charge des victimes de violences sexuelles à destination des professionnel.le.s de la santé et de la justice. Actes du Colloque «Viols et violences sexuelles ». Bruxelles, 26.04.2014.

Delmoitiez B. Abuseurs sexuels et styles d'attachement: mise au point d'un procédé thérapeutique et propositions thérapeutiques cognitivo-comportementales. Mémoire de licence en sciences psychologiques, UCL, 2005.

Dorais M. Nouvelles réalités et nouveaux questionnements dans la prévention des agressions sexuelles. Communication présentée au $8^{e}$ Congrès International Francophone sur l'Agression Sexuelle. Charleroi, juin 2015.

Étude des opinions et des comportements de la population belge en matière de violences sexuelles. Menée pour Amnesty International et SOS Viol, Synthèse presse, 2014. 
Finkelhor D. What's Wrong with Sex between Adults and Children? American Journal of Orthopsychiatry, 1979, 49, (2) : 692-697.

Fischer D. G., McDonald W. L. Characteristics of Intrafamilial and Extrafamilial Child Sexual Abuse. Child Abuse and Neglect, 22, (9), 1998 : 915-929.

Foucault M. Histoire de la sexualité, Tome 1, La volonté de savoir. Gallimard, Paris, 1976.

François I. Comment reconnaître une maltraitance sexuelle récente chez l'adulte et la personne âgée? . In $7^{e}$ Conférence de consensus de la Fédération Française de Psychiatrie, Conséquences des maltraitances sexuelles. Les reconnaître, les soigner, les prévenir, 2003. http://psydoc-

fr.broca.inserm.fr/conf \%26rm/conf//confvictime/experthtml/francois.html

Fuller A. K. Child Molestation and Pedophilia. An Overview for the Physician. JAMA, 261, (4), 1989 : 602-606.

Gabel M. L'enfant abusé sexuellement. Perspectives psychiatriques, 14, (4), 1988 : 251-255 ; Fédération Française de Psychiatrie. Conférence de consensus 22,23 novembre 2001, Paris, Hôpital de la Salpêtrière. Psychopathologie et traitements actuels des auteurs d'agression sexuelle. Fédération Française de Psychiatrie, Paris \& John Libbey Eurotext, Montrouge, 2001.

Gosset D., Hedouin V., Revuelta E., Desurmont M. Maltraitance à enfant. Masson, Paris, 1996.

Haesevoets Y.-H. Devant une révélation de maltraitance sexuelle, émanant d'un enfant ou de ses parents, ou d'un adulte pour lui-même, quels éléments retenir pour la confirmer? $\ln 7^{\mathrm{e}}$ Conférence de consensus de la Fédération Française de Psychiatrie, Conséquences des maltraitances sexuelles. Les reconnaître, les soigner, les prévenir, 2003. http://psydocfr.broca.inserm.fr/conf \%26rm/conf//confvictime/experthtml/haesevoets.html

Haesevoets Y.-H. L'enfant Victime d'inceste. De la séduction traumatique à la violence sexuelle. Réflexion théorico-clinique sur la psychopathologie de l'inceste. De Boeck \& Larcier, Bruxelles, 1997.

Haesevoets Y.-H. Les enfants victimes d'agressions sexuelles et d'inceste : du traumatisme à la déshumanisation. L'abus sexuel de l'enfant, Fonds Houtman, Bruxelles, 1997 : 137-152.

Jones L. M., Finkelhor D., Kopiec K. Why is sexual abuse declining? A survey of state child protection administrators. Child Abuse and Neglect, 2001, 25 : 1139-1158.

Knight R. A., Prentky R. A. Classifying Sexual Offenders. The Development and Corroboration of Taxonomic Models. In Marshall W.L., Laws D.R., Barbaree H.E. (Eds.), Handbook of Sexual Assault. Issues, Theories and Treatment of the Offender. Plenum Press, New York, $1990: 23-54$.

La Fontaine J. S. Child Sexual Abuse and the Incest Taboo : Practical Problems and Theoretical Issues. Man, 23, (1), $1988: 1-16$. 
Lalor K., McElvaney R. Présentation de la nature et de l'ampleur de l'abus sexuel en Europe. La protection des enfants contre la violence sexuelle - une approche globale. Publication de la Réunion interrégionale de haut niveau «La protection des enfants contre la violence sexuelle » co-organisée par la Représentante spéciale du Secrétaire Général des Nations Unies chargée de la question de la violence à l'encontre des enfants et le Conseil de l'Europe, Strasbourg, France, 18-19 juin 2015 : 13-47.

Lamb M. E., Orbach Y., Hershkowitz I., Esplin P. W., Horowitz D. A structured forensic interview protocol improves the quality and informativeness of investigative interviews with children : A review of research using the NICHD Investigative Interview Protocol. Child Abuse \& Neglect, 2007, 31(11-12) : 1201-1231.

Leventhal J. M. A decline in substantiated cases of child sexual abuse in the United States: good news or false hope? Child Abuse and Neglect, 2001, 25 : 1137-1138.

Leventhal J. M. Epidemiology of Sexual Abuse of Children: Old Problems, New Directions. Child Abuse and Neglect, 1998, 22, (6) : 481-491.

Lopez G. Les violences sexuelles sur les enfants. PUF, Paris, 1997.

Lyn T.S., Burton D.L. Adult attachment and sexual offender status. American Journal of Orthopsychiatry, 2004, 74 (2) : 150-159.

Marquet M. Clés pour comprendre la sexualité contemporaine. La Revue Nouvelle, juillet-août $2011: 34-43$.

Marshall W. L., Anderson D., Fernandez Y. Cognitive Behavioural Treatment of Sexual Offenders. John Wiley \& Sons Ltd, Chichester, 1999.

Marshall W. L., Laws D. R., Barbaree H.E. (Eds.) (1990). Handbook of Sexual Assault. Issues, Theories and Treatment of the Offender. Plenum Press, New York, 1999.

Marshall W.L., Laws D.R. (2003). A Brief History of Behavioral and Cognitive Behavioral Approaches to Sexual Offenders : Part 2. The Modern Era. Sexual Abuse : A Journal of Research and Treatment, 2003, 15, (2) :93-120.

Miller-Perrin C. L., Perrin R. D. Child Maltreatment. An Introduction. Sage Publications Inc., Thousand Oaks, 1999.

Miner M. A Striking Government Report Calling for Sexual Health. The Forum, 2001; 13 (2) : $1-6$.

Niveau G., Berclaz M., Lacasa M.-J., With S. Mise en œuvre du protocole d'évaluation de crédibilité SVA dans le contexte médico-légal francophone. Swiss Archives of Neurology ans Psychiatry. 2013; 164 (3) : 99-106.

Office de la Naissance et de l'Enfance. Rapport Annuel 1998.

Organisation Mondiale de la Santé. Report of the Consultation on Child Abuse Prevention, WHO, Geneva 29-31 March 1999. 
Organisation Mondiale de la Santé. www.who.int/topics/sexual health/fr/ , consulté le 30 juin 2015.

Perrone R., Nannini M. Violence et abus sexuels dans la famille. Une approche systémique et communicationnelle. ESF, Paris, 1995.

Proulx J. Les troubles de la personnalité des agresseurs sexuels. In Pham T. (Ed.), L'évaluation diagnostique des agresseurs sexuels. Mardaga, Sprimont, 2006.

Schneider H. Sexual Abuse of Children : Strengths and Weaknesses of Current Criminology, International Journal of Offender therapy and Comparative Criminology, 41, (4), 1997 : 310 324.

Smallbone S.W., Dadds M.R . Further evidence for a relationship between attachment insecurity and coercive sexual behaviour in non-offenders. Journal of Interpersonal violence, 2001, 16 (1) : 22-35.

Smallbone S.W., Dadds M.R. Attachment and coercive sexual behaviour. Sexual Abuse: A Journal of Research and Treatment, 2000, 12 (1) : 3-15.

Summit R. The Child Abuse Accommodation Syndrome. Child Abuse and Neglect, 1983, 7 : 177-193.

Tyler K. A. Social and emotional outcomes of childhood sexual abuse. A review of recent research. Aggression and Violent Behavior, 2002, 7 : 567-589.

Van Gijseghem H. Certains paradoxes dans le traitement socio-judiciaire de l'abus sexuel. L'abus sexuel de l'enfant, Fonds Houtman, Bruxelles, 1997 : 63-72. 